\title{
Markers of Parkinson's Disease Progression Using Cerebrovascular, Autonomic and Small Fiber Polyneuropathy Features
}

\author{
Pimentel DA ${ }^{1,3}$, Ravin $\mathbf{P}^{1}$, Hays $A P^{2}$, Qin $\mathbf{L}^{1}$, Novak $\mathbf{V}^{3}$ and Novak $\mathbf{P}^{1^{*}}$ \\ ${ }^{1}$ Department of Neurology, University of Massachusetts, Worcester, MA, USA \\ ${ }^{2}$ Therapath, New York, NY, USA \\ ${ }^{3}$ Department of Neurology, Beth Israel Deaconess Medical Center, Harvard University, Boston, MA, USA
}

"Corresponding author: Peter Novak MD, Department of Neurology, University of Massachusetts University Campus, S4-810D, 55 Lake Avenue North, Worcester, MA 01655, USA, Tel: 508-334-2527; Fax: 508-856-6778; E-mail: NovakP@ummhc.org

Rec date: Feb 06, 2015, Acc date: Mar 06, 2015, Pub date: Mar 08, 2015

Copyright: ( 2015 Pimentel, et al. This is an open-access article distributed under the terms of the Creative Commons Attribution License, which permits unrestricted use, distribution, and reproduction in any medium, provided the original author and source are credited.

\begin{abstract}
Background: Reliable, quantitative progression markers for Parkinson's disease (PD) are needed. We aimed to determine whether the quantitative analyses of the cerebrovascular, cardiovascular autonomic and small fiber polyneuropathy features correlate with PD severity.
\end{abstract}

Methods: This was a single center, retrospective study. PD patients were evaluated using standardized cardiovascular autonomic reflex testing including deep breathing, Valsalva maneuver, tilt test and skin biopsies for the quantification of epidermal sensory and sweat gland sudomotor fibers. Parkinsonism severity was quantified by the Unified Parkinson'sDisease Rating Scale (UPDRS). Severity of non-motor symptoms was evaluated using QASAT (Quantitative scale for grading of cardiovascular reflex tests, QSART and small fibers densities from skin biopsies) adjusted for age and gender. Correlations were calculated using the Pearson's correlation coefficient ( $r$ ).

Results: We tested 109 PD patients (age $70.14 \pm 11.2$, disease duration $4.8 \pm 4.06$ years). UPDRS-II was correlated with the following QASAT scores: total $(r=0.62, p<0.0001)$, adrenergic function $(r=0.71, p<0.001)$, sensory fiber analysis (epidermal nerve fiber density or ENFD, $r=0.34, p<0.001)$, cerebral blood flow scores $(r=0.43$, $p<0.007)$ and sudomotor function $(r=0.42, p<0.0001)$. UPDRS-III correlated with the following QASAT scores: total $(r=0.46, p<0.0017)$, adrenergic function $(r=0.48, p<0.0001)$, ENFD $(r=0.31, p<0.001)$ and sudomotor function $(r=0.34, p<0.05)$.

Conclusions: Autonomic, cerebrovascular and sensory abnormalities coexist in PD and they progress along with disease severity. The adrenergic score, that primarily measures the duration of orthostatic hypotension during tilt test, had the best correlation with disease severity. QASAT may be a feasible instrument for the objective monitoring of PD progression presenting with autonomic nervous system involvement. These findings need to be validated in a prospective study.

Keywords: Parkinson's disease; Autonomic failure; Autonomic neuropathy; Skin biopsy; QASAT

\section{Abbreviations:}

PD: Parkinson's disease; SFN: Small Fiber Polyneuropathy; OH: Orthostatic Hypotension; UPDRS: Unified Parkinson's Disease Rating Scale; QASAT: Quantitative Scale for Grading of Cardiovascular Autonomic Reflex Tests and Small Fibers from Skin Biopsies; ENFD: Epidermal nerve fiber density; SGFD: Sweat gland fiber density; QSART: Quantitative Sudomotor Axon Reflex Test; HR: Heart rate; BP: Blood pressure; CBF: Cerebral Blood Flow

\section{Introduction}

Parkinson's disease (PD) is the second most common neurodegenerative disease affecting more than 1 million Americans [1]. Autonomic nervous system abnormalities and associated small fiber polyneuropathy (SFN) constitute frequent non-motor complications of PD [2-7].
Autonomic symptom severity correlates with decreased activities of daily living and poor quality of life [8] and may by associated with disease progression in PD [9-11]. Orthostatic hypotension $(\mathrm{OH})$ due to adrenergic failure is the most disabling autonomic symptom of PD. $\mathrm{OH}$ can be identified in about $58 \%$ of $\mathrm{PD}$ patients and is a major risk factor for falls and cognitive decline in this population [12]. PD is associated with a generalized autonomic failure of variable severity that affects all major autonomic branches including the adrenergic, the parasympathetic and the sudomotor functions [6]. Cardiac and generalized sympathetic denervation is observed in the majority of PD patients [7]. The latter suggests that dysautonomia can be associated with SFN affecting both dermal and epidermal fibers [13-15].

The Unified Parkinson's Disease Rating Scale (UPDRS) is the only endorsed marker of disease progression $[16,17]$. However, the UPDRS is a clinical and subjective scale. Therefore, reliable, quantitative and feasible biological progression markers of PD have yet to be identified. These biomarkers should allow to objectively measure disease severity and to track the spectrum of disease at a wide dynamic range. Reliable biomarkers are highly desirable for both medical practice and for 
conducting clinical trials to assess the effect of interventional strategies [17].

In this study we performed a comprehensive evaluation of the cerebrovascular, cardiovascular autonomic and sudomotor functions and obtained epidermal and sweat gland nerve fiber densities (ENDF and SGFD) using skin biopsies from PD patients. These non-motor PD features were correlated with disease severity using the recently validated Quantitative Scale for Grading of Cardiovascular Autonomic Reflex Tests and Small Fibers from Skin Biopsies (QASAT)[18]. QASAT is a quantitative scale that grades abnormalities in domains affected by PD. We hypothesized that 1) QASAT scores correlate with disease severity and 2) that QASAT or a portion of it can be used as a quantitative marker of disease progression. UPDRS was used as the gold standard, as recommended [16,17].

\section{Materials and Methods}

\section{Participants}

This retrospective, single-center study included PD patients who were referred for autonomic function testing to the University of Massachusetts Autonomic laboratory because they had symptoms of autonomic failure. Symptomatic autonomic failure was defined as having at least one of the following: orthostatic lightheadedness, urinary problems (increased urinary frequency or incontinence), signs of gastroparesis, constipation, diarrhea, male sexual dysfunction, cold or hot intolerance and/or sweating abnormalities. PD was clinically defined by a combination of resting tremor, bradykinesia, and rigidity [19].

All subjects had well-defined beneficial response to parkinsonian medications. None of the subjects had a history of prominent hallucinations or other signs suggestive of diffuse Lewy body dementia. None of our participants had cerebellar syndrome, which can be a clue of multiple system atrophy. Subjects presenting comorbidities affecting the autonomic nervous system, such as diabetic and nondiabetic peripheral polyneuropathies, were excluded from the study.

Parkinsonism was evaluated by the Unified Parkinson Disease Rating Scale (UPDRS) part II (activities of daily living) and part III (motor functions) at the "on" state.

Autonomic testing was performed in "on" state. PD medications including levodopa and dopamine agonists were allowed. Medications that affect autonomic function, particularly with antiadrenergic or anticholinergic properties, were held for 5 half-lives if patients could tolerate.

This study was approved by the Institutional Review Board of the University of Massachusetts Medical School as a minimal risk study.

\section{Autonomic testing}

Testing was performed following established standards [20] that have been previously described [21]. Cardiovascular reflex tests included deep breathing, Valsalva maneuver and tilt test. Deep breathing was performed at a respiratory rate of 6 breaths per minute during one minute. Valsalva maneuver was performed with an expiratory pressure equal to $40 \mathrm{~mm} \mathrm{Hg}$ for 15 seconds. After recording 10 minutes of supine rest, patients were tilted during 10 minutes. Postganglionic sympathetic sudomotor function was analyzed by quantitative sudomotor axon reflex test (QSART) in the forearm, proximal leg, distal leg, and foot using Q-Sweat machine (WR Medical Electronics, Stillwater, MN). The stimulation current used was $2 \mathrm{~mA}$, the duration of stimulation was 5 minutes and the sweat volume was collected for 10 minutes.

Skin biopsies were performed following recommended standards $[22,23]$. Full thickness skin biopsies were obtained from the proximal thigh at level of the pubis and at the calf $(10 \mathrm{~cm}$ above the lateral malleolus) using a 3-mm circular disposable punch tool. Samples were immediately transferred into $2 \%$ paraformaldehyde-lysine-periodic acid fixative and processed at Therapath, New York, NY. Samples were immunoperoxidase-stained with the panaxonal marker PGP 9.5 and also stained with hematoxylin/eosin and Congo red to identify skin epidermal/dermal structures, inflammation, vasculitis or amyloidosis. ENFD and SGFD were analyzed using bright microscopy. Normative data has been published [18].

Test results were graded using the QASAT [18]. QASAT is an objective instrument for the grading of dysautonomia, associated small fiber polyneuropathies and cerebral blood flow [18]. QASAT grades standardized cardiovascular reflex tests, QSART, ENFD and SGFD; grading is adjusted for age and gender. QASAT has the following sections: heart rate (HR; includes supine heart rate, heart rate response to tilt, and heart rate variability), blood pressure (BP; supine and tilt induced changes including orthostatic hypotension/hypertension), cerebral blood flow (CBF; both baseline and tilt-induced changes), sudomotor (using QSART and SGFD) and sensory (using ENFD). Adrenergic functions modulate $\mathrm{BP}$ responses to tilt test and to Valsalva maneuver [20]. Therefore the QASAT-adrenergic function score was defined as a summation of orthostatic hypotension during tilt test (QASAT item 7) and Valsalva maneuver - blood pressure (QASAT item 8) scores. QASAT assigns a number to each test result that is proportional to the severity of findings. Normal test results correspond to 0 , while abnormal results are $>0$. Higher scores indicate more severe impairment. For example, BP has a range of 0-12 while total QASAT score is $0-40$. QASAT scores are generated automatically.

\section{Statistical analysis}

Multiple regression analysis and Pearson's correlation coefficient (r) were performed between continuous variables. One-way analysis of variance (ANOVA) was used to test the overall difference between normal, mild, moderate, severe and marked QASAT scores. The post hoc Tukey test was used for pair-wise comparisons if ANOVA results were significant. All statistical analyses were performed using JMP 10.1 (Cary, NC) statistical software.

\section{Results}

A database search showed 118 PD patients that completed autonomic testing. Nine patients were excluded from the analysis because they were taking medications that interfere with autonomic functions. A total 109 patients were included in this study. The characteristics of our PD cohort are listed in Table 1.

QASAT scores were: $17.33 \pm 8.9$ total, $1.7 \pm 1.1 \mathrm{HR} ; 5.0 \pm 4.28 \mathrm{BP}$, $5.28 \pm 3.6 \mathrm{CBF}, 1.42 \pm 1.0$ sudomotor function (QSART); $0.87 \pm 1.3$ SGFD and $1.27 \pm 0.13$ ENFD.

Table 2 shows the correlation between QASAT and parkinsonian variables. UPDRS-II was positively correlated with total, adrenergic function, CBF, sudomotor-QSART and ENFD scores. UPDRS-III was positively correlated with total, adrenergic function, $\mathrm{CBF}$, sudomotor 
function (QSART) and ENFD scores. The Hoehn and Yahr stage of disease was correlated with total, HR, adrenergic function, CBF, sudomotor (QSART) and ENFD scores. No correlations at the $\mathrm{p}<0.05$ level of significance were found between QASAT (total or subscores) and levodopa equivalent dose [24] (LED) or levodopa dose. No correlations at $\mathrm{p}<0.05$ level were found between LED or levodopa and systolic/mean/diastolic BP/CBF velocity either during baseline supine position or during tilt.

\begin{tabular}{|l|l|l|}
\hline Patient characteristics & Mean \pm SD & Range \\
\hline $\mathrm{N}$ & 109 & $\mathrm{NA}$ \\
\hline Age, years & $70.14 \pm 11.2$ & $41-93$ \\
\hline Gender, m/f & $58 / 51$ & $\mathrm{NA}$ \\
\hline BMI, kg/m² & $25.3 \pm 5.37$ & $14.9-41.5$ \\
\hline Hoehn and Yahr stage & $2.1 \pm 0.76$ & $1-4$ \\
\hline Disease duration, years & $4.8 \pm 4.06$ & $0.6-22$ \\
\hline UPDRS II & $9.08 \pm 7.67$ & $0-33$ \\
\hline UPDRS III & $18.28 \pm 13.34$ & $0-71$ \\
\hline LED, mg & $400.4 \pm 263.5$ & $0-1300$ \\
\hline
\end{tabular}

Table 1: Characteristics of Parkinson's disease subjects. LED: Daily Levodopa Equivalent.

\begin{tabular}{|l|l|l|l|}
\hline QASAT & UPDRS-II & UPDRS-III & Hoehn and Yahr \\
\hline Total & $0.62, p<0.0001$ & $0.46, p<0.0017$ & $0.47, p<0.0017$ \\
\hline Heart rate & $0.17, p<0.07$ & $0.18, p<0.06$ & $0.33, p<0.0005$ \\
\hline Adrenergic & $0.71, p<0.001$ & $0.48, p<0.0001$ & $0.44, p<0.001$ \\
\hline $\begin{array}{l}\text { Cerebral blood } \\
\text { flow }\end{array}$ & $0.43, p<0.009$ & $0.36, p<0.07$ & $0.44, p<0.001$ \\
\hline $\begin{array}{l}\text { Sudomotor- } \\
\text { QSART }\end{array}$ & $0.42, p<0.0001$ & $0.34, p<0.05$ & $0.30, p<0.0017$ \\
\hline $\begin{array}{l}\text { Sudomotor- } \\
\text { Sweat glands }\end{array}$ & $0.04, p<0.74$ & $0.08, p<0.08$ & $0.03, p<0.74$ \\
\hline Sensory & $0.34, p<0.003$ & $0.31, p<0.001$ & $0.21, p<0.029$ \\
\hline
\end{tabular}

Table 2: Pairwise correlations using the Pearson's correlation coefficient ( $r$ ) between QASAT scores with UPDRS II, UPDRS III and the Hoehn and Yahr stage of disease.

\section{Discussion}

This study showed that autonomic, cerebrovascular and sensory abnormalities coexist in PD and that they progress with disease severity. The correlation between QASAT-total score and UPDRS-II was moderately strong $(\mathrm{r}=0.62)$. However, there was a strong correlation between QASAT-adrenergic function scores and UPDRSII $(\mathrm{r}=0.71)$. QASAT-total and/or QASAT-adrenergic function scores may be objective and fully quantitative markers of disease severity, considering that UPDRS (clinical gold standard of disease progression) is biased towards the assessment of motor deficits [25]. Therefore, UPDRS might underestimate autonomic features, particularly in early and advanced PD stages [26] (Figure 1).

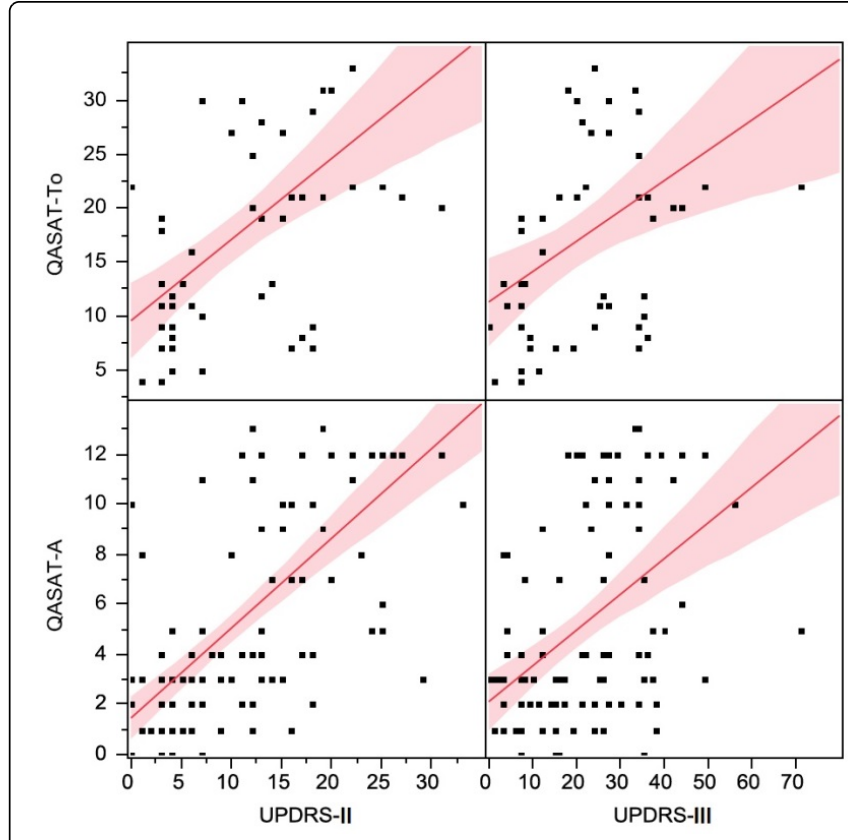

Figure 1: Correlation between QASAT total and adrenergic function scores with UPDRS II and III. To: Total score; A: Adrenergic function score. Each dot corresponds to one patient.

QASAT-adrenergic function scores primarily measure the duration of $\mathrm{OH}$ during tilt test and Valsalva maneuver-induced BP responses. This study showed that $\mathrm{OH}$ is not a yes or no phenomenon. Rather, it has a continuous severity gradient ranging from none to a severe progressive BP drop associated with reduced cerebral perfusion during tilt test. Valsalva maneuver is the most sensitive test to detect milder forms of adrenergic failure [27]; however, the tilt test is more appropriate to detect advanced disease. It should be emphasized that tilt test-induced $\mathrm{OH}$, as defined in QASAT, differs from the definition of $\mathrm{OH}$ used in most of population studies. Traditionally, $\mathrm{OH}$ is defined as a BP drop at minute 3 of active standing. QASAT uses the passive standing, e.g. tilt test, and measures $\mathrm{BP}$ every minute throughout the study [18].

Adrenergic failure occurs primarily due to a generalized impairment of sympathetic-mediated vasoconstriction [28]. Since the adrenergic domain had the best correlation with PD severity, it could be disproportionately more affected in PD compared to other autonomic domains. Cardiovascular reflex testing cannot differentiate between the central (e.g. preganglionic) and peripheral (e.g. postganglionic) components of sympathetic impairment. Pathological studies show degeneration in both the central and the peripheral autonomic nervous systems including autonomic centers in the brainstem [29], and sympathetic pre- and postganglionic neurons [29]. Lewy bodies were also found in the adrenergic nerves that innervate blood vessels in the dermis of patients with PD [30]. Cardiac imaging studies [8] show generalized sympathetic denervation in $\mathrm{PD}$ with $\mathrm{OH}$. These findings indicate that adrenergic failure in PD occurs due to mixed pre- and postganglionic degeneration. None of the QASAT scores correlated with levodopa or dopaminergic medications; this suggests that QASAT grading is unrelated to pharmacologic treatment. 
Although QASAT may be a sensitive tool for tracking autonomic dysfunction progression, a disadvantage is its nonspecificity. A recent validation study [18] showed that QASAT can distinguish MSA from $\mathrm{PD}$, but it cannot distinguish diabetes from PD. However, QASAT was not designed as a diagnostic tool, but as a tool that can track the progression of dysautonomia and associated small fiber polyneuropathy.

Nevertheless, our study indicates that QASAT may be a useful surrogate of PD progression. The main advantage of QASAT is that it is objective, fully quantitative and automatically generated, hence it can complement UPDRS. Furthermore, QASAT is modular, hence only a portion of it can be used. For example, QASAT-cardiovascular might be sufficient to track autonomic functions.

Major shortcomings of this study are its retrospective nature and a subject selection biased towards dysautonomia. Therefore, these results need to be validated preferentially in a prospective study to confirm that these results can be extrapolated to all PD patients.

In conclusion, $\mathrm{PD}$ is associated with cerebrovascular, autonomic and sensory abnormalities that coexist and progress with disease severity. QASAT may be a valuable tool for tracking PD disease severity.

\section{Acknowledgements}

The authors thank Shane Stanek, Donald Chin and Lina Garcia for their help in data collection. This study was funded by Department of Neurology, University of Massachusetts.

\section{References}

1. De Lau LML, Breteler MMB (2006) Epidemiology of Parkinson's disease. Lancet Neurol 6: 525-535.

2. Simuni T, Sethi K (2008) Nonmotor manifestations of Parkinson's disease. Ann Neurol: S65-80.

3. Braak H, de Vos RAI, Bohl J, Del Tredici K (2006) Gastric alphasynuclein immunoreactive inclusions in Meissner's and Auerbach's plexuses in cases staged for Parkinson's disease-related brain pathology. Neurosci Lett 1: 67-72.

4. Djaldetti R, Lev N, Melamed E (2009) Lesions outside the CNS in Parkinson's disease. Mov Disord Off J Mov Disord Soc 6: 793-800.

5. Lipp A, Sandroni P, Ahlskog JE, Fealey RD, Kimpinski K, et al. (2009) Prospective differentiation of multiple system atrophy from Parkinson disease, with and without autonomic failure. Arch Neurol 6: 742-750.

6. Thaisetthawatkul P, Boeve BF, Benarroch EE, Sandroni P, Ferman TJ, et al. (2004) Autonomic dysfunction in dementia with Lewy bodies. Neurology 10: 1804-1809.

7. Sharabi Y, Imrich R, Holmes C, Pechnik S, Goldstein DS (2008) Generalized and neurotransmitter-selective noradrenergic denervation in Parkinson's disease with orthostatic hypotension. Mov Disord Off J Mov Disord Soc 12: 1725-1732.

8. Gallagher DA, Lees AJ, Schrag A (2010) What are the most important nonmotor symptoms in patients with Parkinson's disease and are we missing them? Mov Disord Off J Mov Disord Soc 15: 2493-2500.

9. Shindo K, Iida H, Watanabe H, Ohta E, Nagasaka T, Shiozawa Z (2008) Sympathetic sudomotor and vasoconstrictive neural function in patients with Parkinson's disease. Parkinsonism Relat Disord 7: 548-552.

10. Kim JB, Kim B-J, Koh S-B, Park K-W (2014) Autonomic dysfunction according to disease progression in Parkinson's disease. Parkinsonism Relat Disord 3: 303-307.
11. Maetzler W, Karam M, Fruhmann Berger M, Heger T, Maetzler C, et al. (2015) Time- and frequency-domain parameters of heart rate variability and sympathetic skin response in Parkinson's disease. J Neural Transm Vienna Austria 1996 3: 419-425.

12. Allcock LM, Ullyart K, Kenny RA, Burn DJ (2004) Frequency of orthostatic hypotension in a community based cohort of patients with Parkinson's disease. J Neurol Neurosurg Psychiatry 10: 1470-1471.

13. Dabby R, Djaldetti R, Shahmurov M, Treves TA, Gabai B, et al. (2006) Skin biopsy for assessment of autonomic denervation in Parkinson's disease. J Neural Transm Vienna Austria 1996 9: 1169-1176.

14. Novak P, Marya NB, Whren K, Bhawan J (2009) Dermal sheet preparations in the evaluation of dermal innervation in Parkinson's disease and multiple system atrophy. J Cutan Pathol 3: 296-301.

15. Nolano M, Provitera V, Estraneo A, Selim MM, Caporaso G, et al. (2008) Sensory deficit in Parkinson's disease: evidence of a cutaneous denervation. Brain J Neurol Pt 7: 1903-1911.

16. Harrison MB, Wylie SA, Frysinger RC, Patrie JT, Huss DS, et al. (2009) UPDRS activity of daily living score as a marker of Parkinson's disease progression. Mov Disord Off J Mov Disord Soc 2: 224-230.

17. Maetzler W, Liepelt I, Berg D (2009) Progression of Parkinson's disease in the clinical phase: potential markers. Lancet Neurol 12: 1158-1171.

18. Novak P. Quantitative Scale for Grading of Cardiovascular Autonomic Reflex Tests and Small Fibers from Skin Biopsies. J Neurol Disord. Under revision.

19. Hughes AJ, Daniel SE, Kilford L, Lees AJ (1992) Accuracy of clinical diagnosis of idiopathic Parkinson's disease: a clinico-pathological study of 100 cases. J Neurol Neurosurg Psychiatry 3: 181-184.

20. Low PA, Sletten DM (2008) Laboratory evaluation of autonomic failure: Clinical Autonomic Disorders. (3rdedn), Lippincott Williams \& Wilkins, Philadelphia, USA.

21. Novak P (2011) Quantitative autonomic testing. J Vis Exp JoVE 53.

22. Lauria G, Lombardi R, Camozzi F, Devigili G (2009) Skin biopsy for the diagnosis of peripheral neuropathy. Histopathology 3: 273-285.

23. England JD, Gronseth GS, Franklin G, Carter GT, Kinsella LJ, et al. (2009) Practice Parameter: evaluation of distal symmetric polyneuropathy: role of autonomic testing, nerve biopsy, and skin biopsy (an evidence-based review). Report of the American Academy of Neurology, American Association of Neuromuscular and Electrodiagnostic Medicine, and American Academy of Physical Medicine and Rehabilitation. Neurology 2: 177-184.

24. Tomlinson CL, Stowe R, Patel S, Rick C, Gray R, Clarke CE (2010) Systematic review of levodopa dose equivalency reporting in Parkinson's disease. Mov Disord 15: 2649-2653.

25. Martínez-Martín P, Gil-Nagel A, Gracia LM, Gómez JB, Martínez-Sarriés J, Bermejo F (1994) Unified Parkinson's Disease Rating Scale characteristics and structure. The Cooperative Multicentric Group. Mov Disord Off J Mov Disord Soc 1: 76-83.

26. Chaudhuri KR, Odin P, Antonini A, Martinez-Martin P (2011) Parkinson's disease: the non-motor issues. Parkinsonism Relat Disord 10: 717-723.

27. Novak P (2011) Assessment of sympathetic index from the Valsalva maneuver. Neurology 23: 2010-2016.

28. Goldstein DS, Holmes CS, Dendi R, Bruce SR, Li S-T (2002) Orthostatic hypotension from sympathetic denervation in Parkinson's disease. Neurology 8: 1247-1255.

29. Braak H, Sastre M, Bohl JRE, de Vos RAI, Del Tredici K (2007) Parkinson's disease: lesions in dorsal horn layer I, involvement of parasympathetic and sympathetic pre- and postganglionic neurons. Acta Neuropathol (Berl) 4: 421-429.

30. Ikemura M, Saito Y, Sengoku R, Sakiyama Y, Hatsuta H, et al. (2008) Lewy body pathology involves cutaneous nerves. J Neuropathol Exp Neurol 10: 945-953. 